\title{
Modelo SISTÉMICO DEL IMPACTO DEL GAS NATURAL EN EL desarrollo sustentable del Perú*
}

\author{
Rosario Guzmán Jiménez \\ rguzman@ulima.edu.pe \\ Angélica Kamiyama Murakami \\ akamiya@ulima.edu.pe \\ Universidad de Lima
}

\section{Resumen}

El gas natural es considerado el combustible del siglo XXI, por ser el más limpio entre los combustibles fósiles, por tener un precio competitivo y por la facilidad de su suministro. Para muchos el gas natural de Camisea constituye la oportunidad de impulsar el desarrollo económico y social del Perú. Sin embargo, hasta el momento no existe una política clara sobre su destino. La formulación de una política inadecuada podría resultar en el desaprovechamiento y el agotamiento de este valioso recurso sin haber contribuido a lograr las metas del desarrollo nacional. Este artículo presenta una visión sistémica de la compleja red de valor del gas natural en forma de un diagrama causal, resultado del análisis de las dimensiones energética, económica, social y medio ambiental del desarrollo sustentable. Una vez que este modelo sea implementado con una herramienta de software, podrá ser utilizado para analizar el impacto que diversas políticas sobre el gas natural tendrían en el desarrollo del país.

\section{Palabras clave:}

Gas natural, cadena de valor, pensamiento sistémico, desarrollo sustentable

* Este artículo no hubiera sido posible sin el apoyo del Instituto de Investigación Científica de la Universidad de Lima (IDIC) que auspició el proyecto "Sistema de Cadena de Valor del Gas Natural en el Perú" realizado por las autoras. Del mismo modo, agradecen sinceramente el aporte invalorable del doctor Luis Rabelo y de los ingenieros Percy Castillo, Luis Espinoza y Bruno Paulsen; asimismo, a todos aquellos que de alguna manera las apoyaron en el desarrollo del presente artículo. 


\section{Introducción}

En el nuevo milenio la humanidad se enfrenta al gran reto de satisfacer la creciente demanda de energía para sustentar el crecimiento económico y poblacional, y al mismo tiempo cuidar la calidad del medio ambiente. La energía es requisito para el desarrollo, pero también es una de las principales causas de deterioro del medio ambiente, puesto que la mayor parte de las necesidades de energía se cubren con combustibles fósiles cuyas emisiones están contribuyendo al cambio climático y afectando la calidad del aire. Por otra parte, un tercio de la población mundial no tiene acceso a servicios modernos de energía y depende de los combustibles baratos pero sumamente contaminantes. Mientras se espera un mayor desarrollo de fuentes más limpias de energía y de políticas de conservación de energía, el gas natural surge como una importante solución transitoria, dado que es menos contaminante que el petróleo y el carbón, es todavía abundante y es más económico [1].

En el país existen reservas importantes de gas natural, como los yacimientos de Camisea, que podrían contribuir al desarrollo sustentable del país. Existen dos posiciones con respecto al desarrollo del gas: la posición pro exportadora, que sostiene que las reservas exceden a la demanda interna y que por lo tanto se justifica el proyecto de exportación de gas; la otra posición sostiene que exportando el gas natural se desperdiciaría la oportunidad de utilizarlo para lograr el desarrollo del país.

El pensamiento sistémico es un enfoque para analizar sistemas complejos que permiten ver el impacto que diferentes políticas y decisiones pueden tener sobre el comportamiento global del sistema [2]. Un análisis de la problemática del gas natural aplicando el pensamiento sistémico podría aportar claridad en esta controversia y señalarnos el camino adecuado para lograr el desarrollo sustentable.

\section{Usos y ventajas del gas natural}

El gas natural es un combustible constituido por una mezcla de hidrocarburos livianos cuyo componente principal es el metano ( $\mathrm{CH} 4)$; es limpio, sin color, sin olor y más ligero que el aire. Es un combustible fósil como el petróleo y el carbón, formado principalmente de restos de plantas, animales y microorganismos que vivieron hace millones de años y yacieron sepultados bajo la superficie de la tierra a altas presiones y temperaturas. El gas natural es entonces extraído del subsuelo y es transportado y distribuido mediante ductos a los centros de consumo. 
El gas natural es una fuente de energía versátil, cuya aplicación más frecuente es la generación de calor, ya sea en el hogar, el comercio, la industria y en la generación de energía eléctrica mediante la cogeneración y la generación eléctrica con ciclos combinados. El gas natural tiene también importantes usos en la industria petroquímica como materia prima para la fabricación de una variedad de productos, como plásticos y fertilizantes. Otro importante uso es como combustible para vehículos [3].

En adición a la versatilidad en el uso, el gas natural tiene beneficios ambientales. El gas natural es el combustible fósil que menos contamina el ambiente, debido a que es el que produce menos dióxido de carbono y otras emisiones a la atmósfera, aparte de que no emite partículas sólidas ni cenizas en su combustión. El gas natural, por lo tanto, contribuye a la disminución del efecto invernadero. Queda claro que la sustitución del petróleo y sus derivados por el gas natural produce significativos beneficios no solo en el plano económico sino también en el plano ambiental [1].

\section{De la cadena de valor del gas natural a una red de valor del gas natural}

La cadena de valor es un concepto introducido por Michael Porter [4], que está enfocado en el conjunto de factores y relaciones entre los distintos procesos de la empresa. Abarca desde el suministro de la materia prima, recorriendo las distintas actividades hasta la entrega de un bien final que aporta un valor al cliente; se observan dos tipos de actividades: las primarias, referidas al flujo físico de creación de valor, y las secundarias, o de apoyo, orientadas a actividades de gestión en el nivel intraorganizacional, tal como se aprecia en la figura 1.

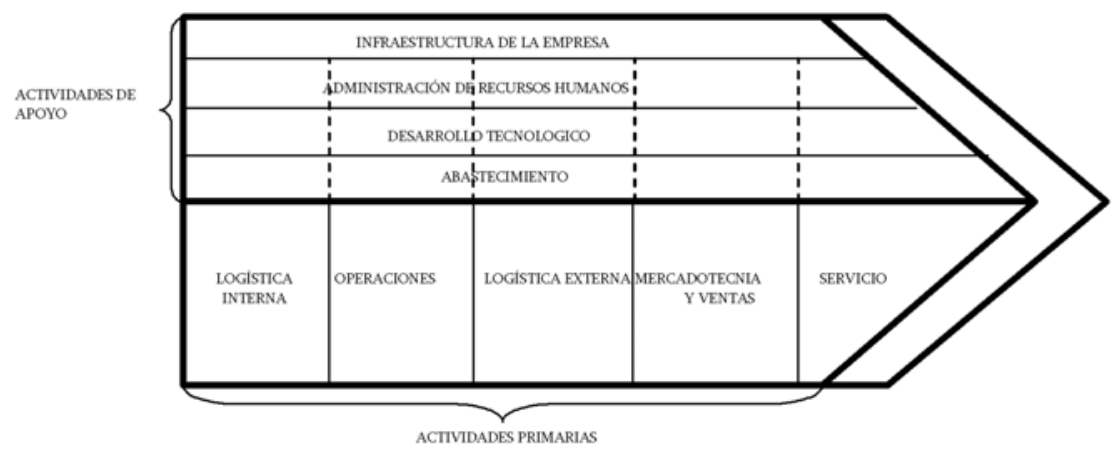

Figura 1. Cadena de Valor, Michael Porter, 1990. 
Su aplicación en el mundo real originó posteriormente el concepto de cadena de valor interorganizacional, que también es conocido como sistema o red de valor que se visualiza en la figura 2, donde se aprecia una red estratégica de organizaciones o empresas independientes: proveedores, productores, distribuidores y compradores. En una red de valor las empresas reconocen la necesidad de trabajar juntas para identificar objetivos estratégicos y están dispuestas a compartir riesgos y beneficios e invertir tiempo, energía y recursos a través de un trabajo articulado. El propósito primario de la red de valor es responder con más efectividad y eficiencia a las necesidades del mercado a través de la cooperación, comunicación, y coordinación entre las empresas.

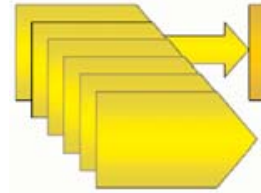

Cadena de valor de proveedores

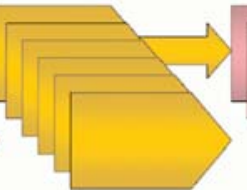

Cadena de valor del sector
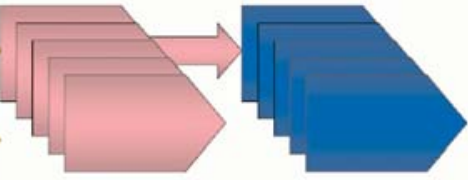

Cadena de valor Cadena de valor de distribuidoresde compradores

Figura 2. Cadena de valor interorganizacional, Michael Porter, 1990

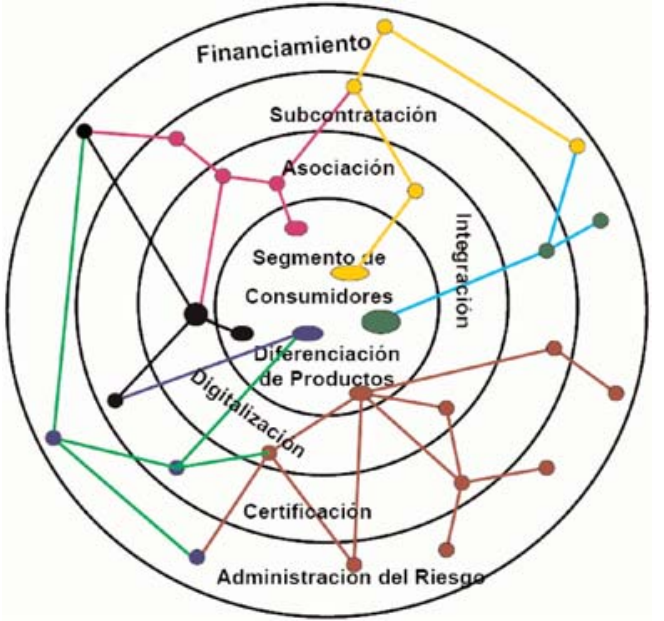

Figura. 3. Red de Valor, Vijay Gurbaxani, 2002

Vijay Gurbaxani [5] define el concepto de una red de valor como una colaboración integrada de empresas especializadas, en la que cada una ofrece servicios y bienes complementarios. El fin es crear y comercializar productos finales y servicios para el cliente; adicionalmente las redes de valor permiten a las organizaciones explotar las economías de escala, que se obtienen por formar parte de una estructura más grande. 
En el caso del gas natural, los yacimientos se encuentran generalmente lejos de los centros de consumo; luego de ser extraído del subsuelo el gas natural es transportado a los centros de consumo mediante gasoductos, y distribuido a los usuarios finales mediante redes de distribución. Estas actividades de producción, transporte y distribución conforman entonces la cadena de valor del gas natural.

La etapa de producción comprende las operaciones de exploración, extracción, tratamiento del gas extraído del subsuelo y separación del gas natural seco y los líquidos del gas natural. La etapa de transporte consiste en la conducción del gas natural producido hasta los centros de consumo, principalmente por gasoductos a alta presión; también se le transporta como gas natural licuado (GNL) en buques metaneros y camiones criogénicos, o como gas natural comprimido (GNC) en cilindros de alta presión. La etapa de distribución consiste en el reparto de gas natural a los puntos de consumo mediante ductos subterráneos, a media y baja presión.

En el proceso de identificación de valor en la cadena del gas natural se analizó el discurso de René Maheu, director general de la Unesco en la Conferencia Intergubernamental sobre los aspectos institucionales, administrativos y financieros de las políticas culturales [6]. Una parte del discurso dice: "El hombre es el medio y el fin del desarrollo; no es la idea abstracta y unidimensional del Homo Economicus, sino una realidad viviente, una persona humana, en la infinita variedad de sus necesidades, sus posibilidades y sus aspiraciones... Por consiguiente, el centro de gravedad del concepto de desarrollo se ha desplazado de lo económico a lo social, y hemos llegado a un punto en que esta mutación empieza a abordar lo cultural".

En consecuencia, la aspiración de una ventaja competitiva para el país a través de la red de valor del gas natural debería estar alineada a la propuesta del desarrollo sustentable, por lo que se requiere de un modelo que permita analizar las interrelaciones de todos los elementos de la red de valor y el impacto que las estrategias de desarrollo ejercerían sobre cada uno de los elementos de dicha red. 


\section{Antecedentes}

\section{Panorama internacional y regional del gas natural}

El gas natural se perfila como la fuente de energía primaria con mayor crecimiento de demanda a nivel mundial después del carbón [7], [8]. Se estima que el consumo del gas natural se incrementará en un promedio anual de $2,4 \%$ entre el 2003 y el 2030, y se espera que sea la alternativa elegida por sus beneficios ambientales en varias partes del mundo. Su participación en el consumo total de energía crecería de $24 \%$ en el 2003 a $26 \%$ en el 2030, de 95 TCF $^{1}$ a 182 TCF. En el 2003, la demanda de gas natural de los países de la Organización para el Desarrollo y la Cooperación Económicos $(\mathrm{ODCE})^{2}$ representó aproximadamente la mitad de la demanda mundial. Sin embargo, su incremento promedio anual proyectado de demanda es de 1,5\% mientras que el promedio proyectado para los países que no pertenecen a la ODCE es de 3,3\%.

Las reservas mundiales de gas natural tienden a aumentar y a inicios del 2006 las reservas probadas mundiales eran de aproximadamente 6.112 TCF. Casi tres cuartas partes de las reservas mundiales se encuentran en Medio Oriente y Europa del Este. Entre Rusia, Irán y Qatar poseen el $58 \%$ de las reservas mundiales. Se estima en 4.221 TCF las reservas mundiales de gas natural aún no descubiertas.

Los países europeos que no son miembros de la ODCE y los países de Europa del Este y del Medio Oriente solo producen el 39\% de la producción mundial, a pesar de contar con el $75 \%$ de las reservas mundiales. En el 2003, los países de la ODCE produjeron el $41 \%$ de la producción mundial mientras que su consumo representó el $52 \%$ de la demanda mundial. Se proyecta un incremento anual de solo $0,5 \%$ en su producción, mientras que su demanda se estima que crecerá a un ritmo de 1,5\% anual. En consecuencia, estos países dependerán cada vez más de las importaciones de gas natural en la forma de gas natural licuado (GNL). Actualmente los países de la ODCE cubren más de un tercio de sus requerimientos energéticos con gas natural importado. Se estima que en el 2030 ese porcentaje será de $22 \%$. Se espera que el GNL se convierta en una importante forma de suministro para atender las demandas mundiales. Igualmente, se estima que la producción de gas natural en las economías emergentes del Asia no abastecería totalmente el crecimiento proyectado de la demanda, requiriéndose de la importación del gas natural.

1. Terapies cúbicos o $10^{12}$ pies cúbicos.

2. ODCE, organización internacional intergubernamental que reúne a los países más industrializados de economía de mercado. 
En América del Sur y América Central se espera que el gas natural sea la fuente de energía de máximo crecimiento, con un incremento anual proyectado en 3,9\%, de 3,8 TCF en el 2003 a 10,8 TCF en el 2030. En la región, Trinidad y Tobago ya exporta fuera de la región mientras que los otros países exportadores del gas natural la exportan a través de los gasoductos que conectan a Bolivia, Brasil, Argentina, Chile y Uruguay. Existen propuestas para construir gasoductos que conecten al Perú con Ecuador y Chile, Venezuela con Colombia y Brasil, y Colombia con Panamá. Estos nuevos gasoductos serían posteriormente conectados a los ya existentes para formar una red sudamericana de gas natural. Se estima que el Perú comenzará a exportar en el 2010.

En general, el sector de consumo final con los mayores incrementos sería el sector eléctrico. Los incrementos en el consumo mundial de gas natural incentivarían el aumento en las inversiones, tanto en exploración y explotación de este combustible como en el desarrollo de gasoductos entre países. Asimismo, de acuerdo con la geopolítica entre productores y consumidores, se requerirán inversiones para la construcción de plantas regasificadoras de GNL.

\section{Panorama energético nacional}

En el Perú existen cinco empresas estatales que representan el 35\% del total de la capacidad de generación eléctrica, mientras 15 empresas privadas representan los restantes 65\%. En términos de distribución, cinco empresas privadas representan juntas el $71 \%$ de la electricidad distribuida en el Perú y 16 empresas estatales proporcionan los $29 \%$ restantes.

La cobertura de la distribución de electricidad ha aumentado a un 75\% hasta el 2005, pero en comparación con otros países en América Latina es aún una cifra baja. La falta de energía eléctrica es más grave en las zonas rurales, en donde aproximadamente el $68 \%$ de la población no tiene electricidad. Al mismo tiempo, la demanda anual de electricidad en el país se incrementa entre 5 y $6 \%$. En un contexto de altos precios de petróleo, el curso indicado para el Perú sería promover el uso del gas natural en el sector industrial, residencial, vehicular y eléctrico [9].

El proyecto Camisea [10] es el más grande proyecto energético en el Perú. Los yacimientos del proyecto están conformados por los campos San Martín y Cashiriari, más conocidos como Bloque 88, localizados en la selva de la región Cusco. Se estima un nivel total de reservas proba- 
das y probables de 11 TCF y 482 millones de barriles de líquidos de gas natural. Los yacimientos de Camisea fueron descubiertos entre 1983 y 1987 por la empresa Shell, pero recién se suscribieron los contratos para el desarrollo del proyecto en el 2000.

Las operaciones de explotación están a cargo del consorcio liderado por la empresa PlusPetrol. El consorcio Transportadora de Gas del Perú, liderado por la empresa Techint, construyó y opera el gasoducto de gas natural y el poliducto de líquidos del gas natural para llevar la producción a Lima y a la planta de fraccionamiento en Paracas, respectivamente.

El proyecto Camisea suministra gas natural desde agosto del 2004 para la demanda interna que aún es incipiente. Basándose en un probable escenario de reducida demanda interna de gas natural, se ha iniciado la ejecución de un proyecto de exportación de gas natural que consiste en la explotación del Bloque 56, aledaño al Bloque 88. Hunt Oil lidera el consorcio Perú $L N G$, que ha empezado la construcción de un terminal de exportación en Pampa Melchorita, a unos $170 \mathrm{~km}$ al sur de Lima. Esa producción será enviada a la costa oeste de Estados Unidos y México, aunque también se vislumbran las posibilidades de su exportación a Chile.

Desde que empezó a abastecer al mercado peruano el proyecto ha tenido varios problemas, sobre todo con rupturas en los tubos que transportan el gas líquido, pero también por la baja demanda interna. A pesar de las ventajas económicas, muy pocas empresas han firmado contratos de suministro de gas. Asimismo, el número de conexiones en las viviendas está creciendo aún más lentamente, y menos de mil viviendas estaban conectadas en octubre del 2005. Esta situación demuestra que la red de valor del gas natural en el Perú aún no ha logrado alcanzar una ventaja competitiva para el país.

\section{Desarrollo sustentable}

\section{Evolución del concepto de desarrollo sustentable}

El pensamiento económico adoptado en los países en vías de desarrollo, por estar impregnado de ideas y teorías económicas elaboradas para países desarrollados, no ha contribuido en el logro del desarrollo esperado. El origen del término desarrollo sustentable se le atribuye a la Conferencia de Naciones Unidas efectuada en Estocolmo en 1972, donde se 
le emplea por vez primera como un concepto según el cual el desarrollo no solo debe considerar el aspecto económico sino también los aspectos sociales y ambientales.

Posteriormente, el concepto se formaliza y entra en vigencia con la difusión del documento Nuestro futuro común, emitido en la Comisión Mundial del Medio Ambiente y el Desarrollo [11] en 1987, por encargo de la Organización de las Naciones Unidas (ONU), que fuera presidida por la primera ministra de Noruega, Harlem Brundtland. El informe proponía reformas al modelo de crecimiento económico, introduciendo mecanismos que hicieran posible un desarrollo sustentable: "La humanidad cuenta con la habilidad para hacer sostenible el desarrollo, asegurar que él mismo atienda a las necesidades del presente sin comprometer la habilidad de las futuras generaciones por atender sus propias necesidades (...) La Comisión cree que la pobreza generalizada ya no es inevitable (...) el desarrollo sostenible implica atender las necesidades básicas de todos y extender a todos la oportunidad para lograr sus aspiraciones de una vida mejor. Un mundo donde la pobreza es endémica será siempre susceptible a catástrofes ecológicas y de otros tipos".

En 1991 el Programa de las Naciones Unidas para el Medio Ambiente (PNUMA), incluye en el concepto de desarrollo sustentable la necesidad de mejorar la calidad de vida humana sin superar la capacidad de carga de los ecosistemas que la sustentan.

En la Conferencia de Río sobre Medio Ambiente y Desarrollo, en 1992, el desarrollo sustentable se presenta como argumento central del debate, reconociendo la importancia de la problemática ambiental y social que afecta al mundo; de igual forma, ese mismo año, la Comisión Económica para América Latina y el Caribe (CEPAL) [12] conceptualiza el desarrollo sustentable a través del análisis de las dimensiones económica, que incluye la posibilidad de sostener la acumulación (crecimiento hacia el futuro); del medio ambiente, teniendo en cuenta la disponibilidad de los recursos (conformado por la amplia gama de elementos que conforman su entorno natural, contempla su alteración e incertidumbre de los efectos del desequilibrio ocasionado por su explotación en el ámbito local como global); social, vinculado a la calidad de vida de la población (nivel de ingreso de las familias, políticas sociales del país, etc.) y la dimensión política, vinculada a la gobernabilidad y vigencia que aseguren el respeto por los derechos del hombre.

En la Cumbre Mundial sobre el Desarrollo Sustentable, celebrada en Johannesburgo, Sudáfrica, en el 2002, se vuelve a debatir acerca de la necesidad de lograr un desarrollo sustentable en todo el planeta. 
Sunkel [13] aclara que:

... el desarrollo sostenible no se refiere a un estado inmutable de la naturaleza y de los recursos naturales e incorpora una perspectiva de largo plazo en el manejo de los mismos, por lo que ya no se apunta a una explotación de los recursos naturales sino a un manejo de éstos; asimismo enfatiza en la necesidad de la solidaridad hacia las actuales y futuras generaciones y defiende la equidad intergeneracional. De otra parte, defiende la necesidad que la dirección de la inversión y del progreso científico tecnológico estén encaminados a la satisfacción de las necesidades presentes y futuras.

Sin embargo, la imperante necesidad y urgencia de resolver los problemas más apremiantes, como son la pobreza, el desempleo, y las necesidades insatisfechas, hace que se involucre toda la población en el desarrollo sustentable. Es por ello que el Programa de las Naciones Unidas para el Desarrollo (PNUD), desde 1990 propone una nueva concepción del desarrollo, el denominado "desarrollo humano", con un nuevo indicador para la medición de este -Índice de Desarrollo Humano (IDH) - y realiza un análisis anual de diferentes problemas políticas, económicas y sociales y su impacto en el desarrollo humano. En el año 1994 emite un nuevo concepto: "El propósito del desarrollo consiste en crear una atmósfera en que todos puedan aumentar su capacidad y las oportunidades puedan ampliarse para las generaciones presentes y futuras".

\section{Contribución del sistema energético al desarrollo sustentable según la CEPAL [12]:}

Las grandes revoluciones tecnológicas que afectaron las actividades de producción y consumo han estado estrechamente vinculadas a la sustitución entre fuentes energéticas primarias. La energía constituye un elemento esencial para la calidad de vida del ser humano y es consumida en todas las actividades productivas; por lo que la disponibilidad de energía ha tenido un papel central en el proceso de desarrollo de la humanidad. En la figura 4 se observa tanto el subsistema de abastecimiento como de consumo energéticos, así como sus interrelaciones con el medio ambiente natural, la economía y la sociedad en la definición del concepto de desarrollo sustentable. 


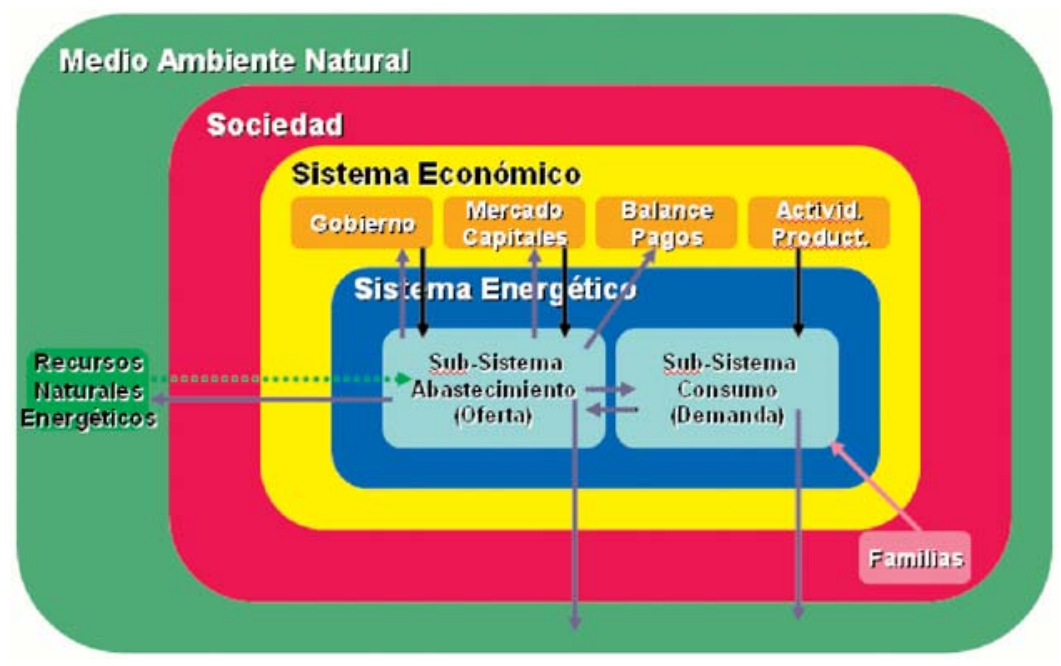

Fig. 4 Desarrollo sustentable según la CEPAL

Asimismo, no hay que olvidar que un manejo no adecuado de los recursos fósiles conduce a un progresivo agotamiento de las reservas correspondientes. Adicionalmente existen múltiples impactos negativos sobre los suelos, el agua y el medio ambiente, que impacta a su vez en la producción, transformación y utilización de la energía.

El Perú no cuenta con una política energética alineada al desarrollo socioeconómico del país, donde todos son protagonistas y destinatarios del desarrollo sustentable, empezando con los poderes del Estado, las autoridades regionales, entidades representativas, trabajadores y el resto de la sociedad.

El presente estudio busca proporcionar los criterios necesarios para el diseño de una política del sector de gas natural, basándose en el enfoque sistémico que permita el análisis del impacto del gas natural en el sistema económico, la sociedad y el medio ambiente.

\section{Modelo del desarrollo sustentable basado en el gas natural}

La dinámica de sistemas ${ }^{3}$ es una metodología para la modelación de sistemas complejos que existen en el mundo real; con ella se pueden ana-

3 La dinámica de sistemas fue creada a principios de la década de 1960 por Jay Forrester, perteneciente al Grupo de Dinámica de Sistemas del MIT (Instituto Tecnológico de Massachussets). 
lizar las complejas relaciones entre los elementos de un sistema. El primer paso de esta metodología consiste en la elaboración del diagrama causal, que es una red en la que aparecen los elementos del sistema y las relaciones entre ellos.

El diagrama causal desarrollado en este estudio ha sido denominado "modelo del desarrollo sustentable". Dicho diagrama causal muestra las distintas relaciones que se dan entre las variables relevantes para el análisis del impacto del gas natural en el desarrollo sostenible del país, el cual considera las dimensiones energética, económica, social y del medio ambiente. Para facilitar la explicación del diagrama causal, inicialmente se dará énfasis a las variables de la dimensión energética y las distintas interrelaciones entre ellas. Luego, se irán incorporando las dimensiones económica, social y del medio ambiente.

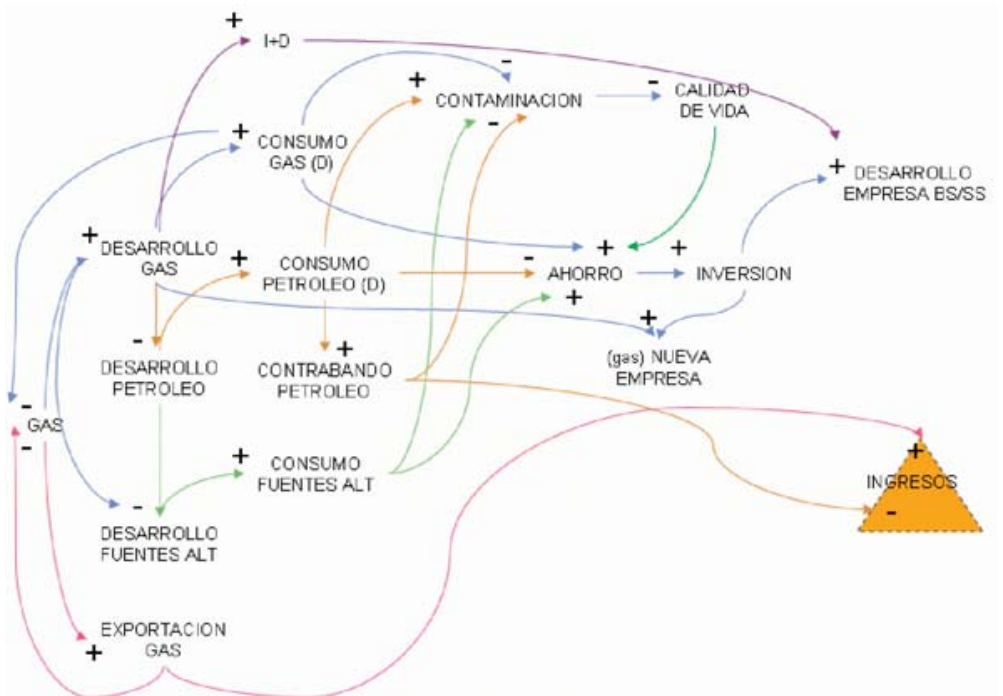

Figura 6. Modelo del desarrollo sustentable: Dimensión energética

\section{La dimensión energética}

El modelo propuesto en la figura 6 muestra al recurso del gas natural como agente dinamizador [13] del desarrollo sustentable. Este recurso puede dinamizar la economía mediante dos estrategias: la primera está dirigida a la exportación, que generaría más ingresos a nivel macroeconómico; la segunda estrategia es la de desarrollo del sector del gas natural dirigido al consumo interno del gas, sustituyendo al petróleo y sus derivados. Debido a que el gas natural es un recurso no renovable, la aplica- 
ción de cualquiera de las estrategias o la combinación de ambas dará como resultado inexorable el agotamiento del recurso; por lo tanto, es necesario considerar el desarrollo de fuentes de energía renovables.

El gas natural es el combustible fósil que menos contamina el medio ambiente; en consecuencia, con un mayor consumo de gas natural, el daño causado al medio ambiente será menor. Varios estudios internacionales han demostrado el impacto negativo en la salud y la economía que origina la contaminación del aire producida principalmente por el sector vehicular [14]. Por otro lado, un mayor consumo de gas natural implicaría un menor consumo de petróleo y derivados, que a su vez desincentivaría el contrabando de estos [15].

\section{La dimensión económica}

En el mercado internacional el precio del gas es menor y más estable que el precio del petróleo. Adecuadas políticas energéticas sobre el gas natural, como la aplicada en Colombia, $[16,17]$ han generado ahorros que luego fueron destinados a la inversión, dinamizándose de esta manera la economía. En nuestro país, existen varias empresas industriales y de generación eléctrica que obtuvieron grandes ahorros con el uso del gas natural [18].

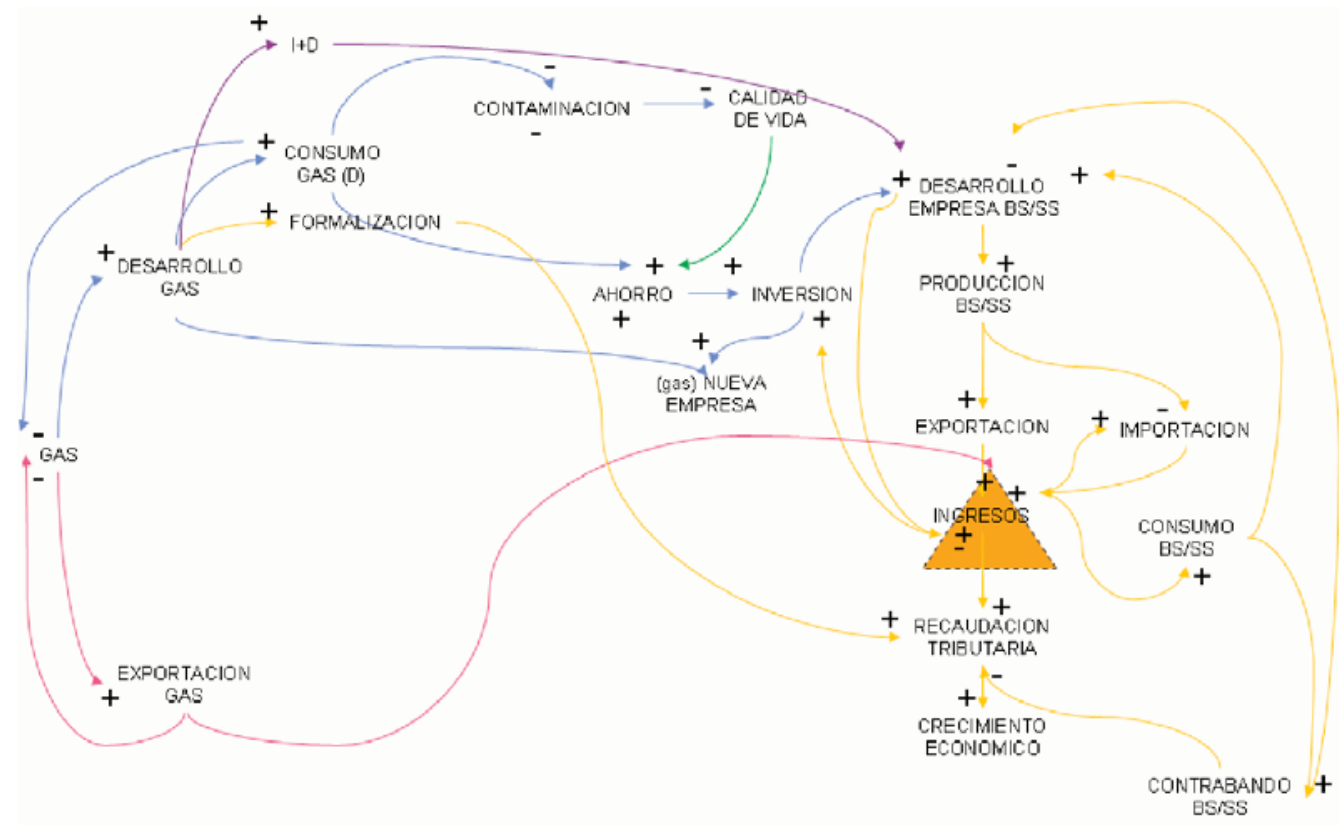

Figura 7. Modelo del desarrollo sustentable: Incorporación de la dimensión económica 
Basándonos en el párrafo anterior, en el modelo se muestra que el consumo de gas generará ahorro, y al haber más ahorro, bajo una política de fomento de producción nacional, se podrían incentivar la inversión en el desarrollo empresarial (modernización de la maquinaria, mejoras u optimización del proceso productivo, etcétera) o para la creación de nuevas empresas (utilizando el gas natural como insumo).

El desarrollo del gas natural también impulsaría la investigación o el desarrollo en las universidades, institutos técnicos o nuevas empresas del sector, tanto en proyectos de desarrollo de nuevas tecnologías, asociadas a la conversión de plantas, como en la incorporación del gas natural en los diferentes sectores: industrial, vehicular, residencial y eléctrico.

La inversión en el desarrollo empresarial incrementará la capacidad de producción y la diversificación de bienes y servicios; una mayor producción incrementará las posibilidades de exportar, lo cual generará ingresos, no solo en el nivel macroeconómico sino también en el microeconómico; además, al producirse mayores ingresos en las empresas, el Estado tendrá una mayor recaudación tributaria; todo ello se reflejaría finalmente en un mayor crecimiento económico.

Si a este incremento en el nivel de producción nacional se suman programas del estado como "Cómprale al Perú"4 se produciría una menor importación de productos que pueden fabricarse en el Perú. Debido a que actualmente existe un alto nivel de importación, se observa la formación de un ciclo entre los niveles de ingresos y los niveles de importación, pero debido a los gastos inherentes al proceso de importación, sumado a los márgenes de ganancia de las empresas que intervienen en el proceso, estos productos importados llegan al ciudadano con precios muy altos o por encima del promedio internacional. Esto ha sido unos de los factores que ha generado el contrabando de productos cuyas consecuencias son la evasión de impuestos y la salida de dinero a otras economías.

A lo anterior se suma el surgimiento de empresas informales que generan una competencia desleal a las empresas formales; el Perú ocupa el tercer lugar en el ranking de informalidad en América Latina y representa un gran problema para el Estado [19]. Los efectos del contrabando y la informalidad han sido bosquejados en la figura 7 y convergen

4 Por Ley 28312 y su Reglamento aprobado por Decreto Supremo No 030 -2005-PRODUCE, se declaró de interés nacional el Programa "Cómprale al Perú", a cargo del Ministerio de la Producción. 
en un impacto negativo en el crecimiento económico del país. Con el gas natural se resuelve el problema de la informalidad, exigiendo a las empresas formalizarse para tener acceso a las ventajas del gas natural [20]. Con el gas natural también se resuelve el problema del contrabando de combustible en el sector vehicular, al haberse puesto en marcha el Sistema de Carga Inteligente de Gas Natural [21], que financia la conversión de los vehículos que utilizan petróleo o gasolina en los que usen gas natural.

\section{La dimensión social}

En la última década se avanzó en la estabilización económica, la pacificación y la reinserción internacional. Y se retrocedió, por el otro lado, con el aumento de la pobreza, la desigualdad, el desempleo y la corrupción, disminuyendo la calidad de vida de millones de peruanos a pesar de los indicadores macroeconómicos positivos [22]. La modernización solo ha beneficiado a ciertas minorías, el desempleo y el subempleo afectan a más de la mitad de la población. Las políticas macroeconómicas y las reformas económicas no han logrado revertir esta situación. Adicionalmente, existe una fragilidad institucional, tanto en el Estado como en las organizaciones de la sociedad civil, lo que genera una elevada dosis de inseguridad colectiva.

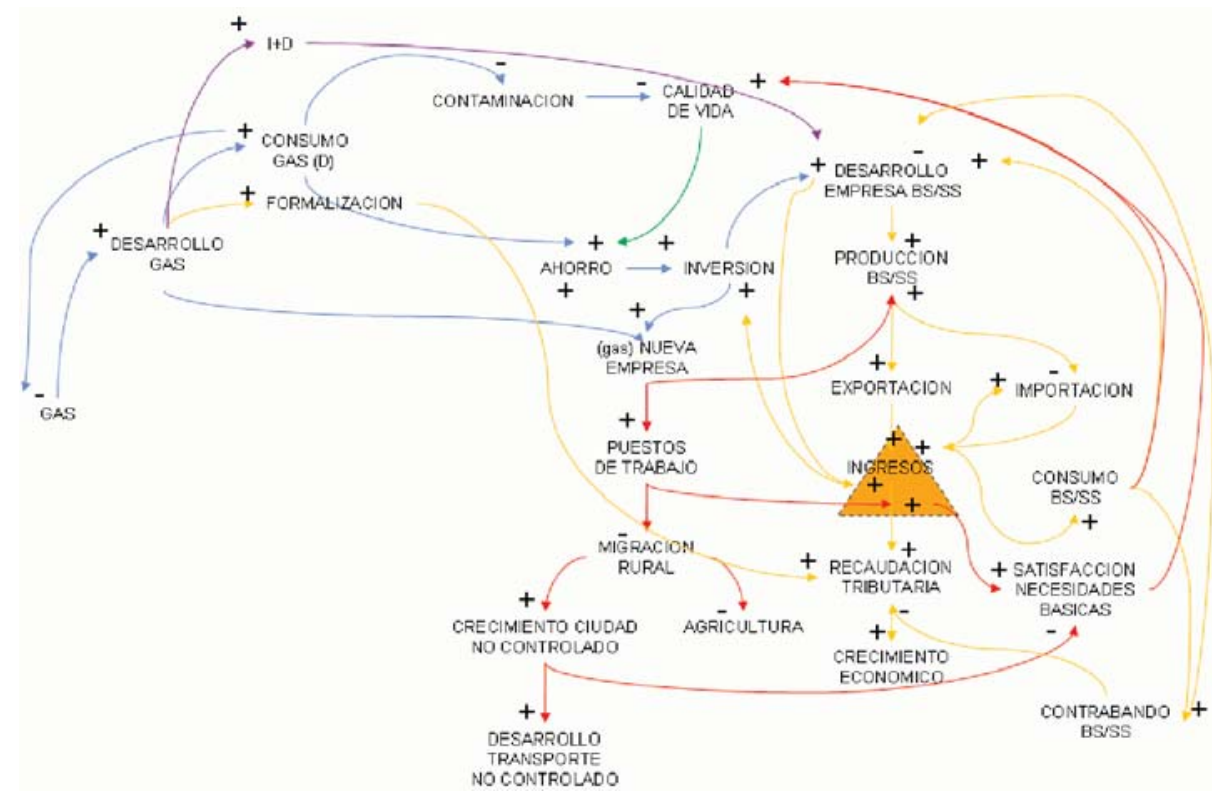

Figura 8. Modelo del desarrollo sustentable: Incorporación de la dimensión social. 
El gas natural como agente dinamizador de la economía lleva al desarrollo empresarial, lo cual a su vez generará más estabilidad, así como la creación de nuevas empresas, creando más puestos de trabajo, y al existir más puestos de trabajo con un sueldo honorable se generarían más ingresos en el nivel microeconómico. Una mayor estabilidad en los ingresos del ciudadano o de mayores ingresos para él, le permitirían satisfacer sus necesidades básicas y a la vez incentivaría un mayor consumo de bienes y servicios; en ambos casos el ciudadano lograría una mejor calidad de vida.

La gestión centralista de los últimos gobiernos y la poca presencia del Estado en los pueblos más apartados ha postergado el desarrollo de esos pueblos, lo que asociado a la poca o casi nula creación de empleos ha generado la migración de la población rural hacia las ciudades más desarrolladas. Las migraciones masivas de las poblaciones rurales a las áreas urbanas han convertido a la mayoría de la población peruana en habitantes urbanos en menos de cuarenta años [23]; ello ha contribuido al crecimiento no controlado de las ciudades, sin una adecuada planificación del proceso de urbanización, ha aumentado el número de asentamientos humanos sin los principales servicios básicos; el hacinamiento, la pérdida de áreas verdes, el crecimiento no controlado del sector vehicular; todo ello deteriora la calidad de vida de los ciudadanos. Estas relaciones causales son bosquejadas en la figura 8.

\section{La dimensión medio ambiental}

El Perú es un país heterogéneo en recursos minerales y biológicos. A escala mundial, es considerado uno de los quince países con mayor biodiversidad; el cuarto país con mayor extensión de bosques tropicales, el primero en cantidad de plantas domesticadas y en número de especies nativas. Sin embargo, el Estado no ha tenido un control adecuado sobre los recursos, por lo que ha habido y existe aún un manejo depredador del medio ambiente natural, que ha llevado a la extinción de muchas especies, la contaminación del aire y del agua, como resultado de un acelerado proceso de urbanización y contaminación ambiental, y que a su vez ha tenido efectos nocivos sobre la salud y calidad de vida, así como cambios irreversibles, que actualmente están afectando las posibilidades de desarrollo futuro del país. 


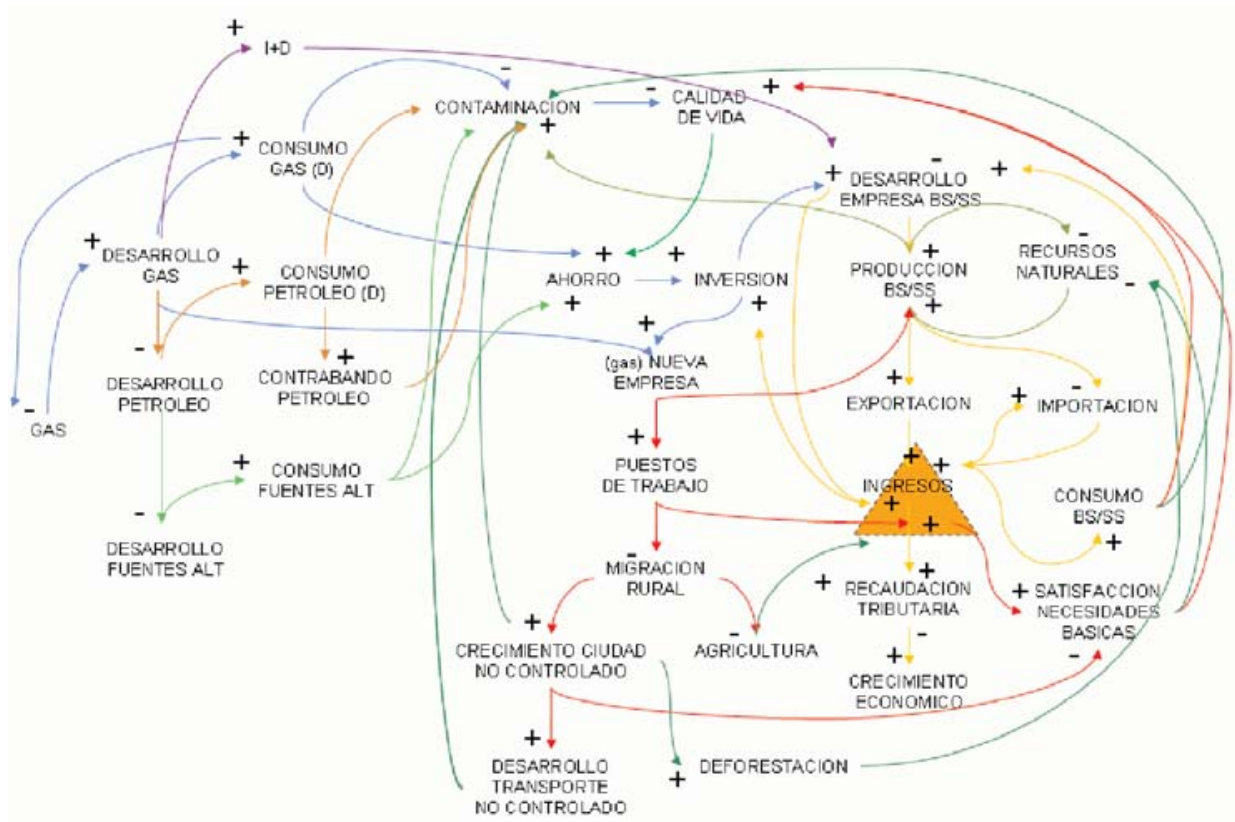

Figura 9. Modelo del gas natural para el desarrollo sustentable: Incorporación, dimensión, medio ambiente

Según el Consejo Nacional del Medio Ambiente, uno de los principales contaminantes del aire en las ciudades proviene del sector vehicular, por el consumo del diesel $2[24,25]$ y del sector industrial (fundiciones, mineras, etcétera). Lima es la ciudad que concentra la mayor población vehicular [26] y presenta niveles de contaminación atmosféricos muy altos, por lo que es considerada una de las ciudades del continente con el aire de peor calidad [27]. Asimismo, muchos estudios internacionales han comprobado el impacto negativo de la contaminación ambiental en la salud ${ }^{5}$ y la economía. En los países desarrollados los efectos en la salud debido a la contaminación del aire cuestan casi $2 \%$ del producto bruto interno por año. En los países en vías de desarrollo el costo es mucho más alto, entre $5 \%$ y $20 \%$ del producto bruto interno [28].

La figura 9 muestra que el crecimiento de las ciudades y el crecimiento del transporte no controlado, así como la pérdida de áreas verdes por el proceso de urbanización contribuyen a un incremento en los niveles de contaminación que perjudica la calidad de vida.

5 Centro Nacional de Investigación de Medio Ambiente y Salud en Neuherberg (Alemania), University of Queensland, de Brisbane (Australia). 


\section{Conclusiones}

El uso adecuado del recurso del gas natural puede ser el inicio de la solución a muchos problemas de nuestro país, utilizándolo como herramienta de desarrollo sustentable para todos los peruanos.

La figura 9 integra las cuatro dimensiones en un solo diagrama causal, de modo que el impacto de cualquier estrategia del gas natural sobre el desarrollo sostenible pudiera ser analizado. A partir de este diagrama causal se elaborará el modelo dinámico, que posteriormente será implementado mediante una herramienta informática. Para elaborar el modelo dinámico se desarrollarán las expresiones que describan matemáticamente cada una de las relaciones incluidas en el diagrama causal. Para tal fín se requerirá de la participación de un equipo multidisciplinario, formado por especialistas en energía, economía, sociología y medio ambiente.

Una vez validado el modelo dinámico, podrá ser utilizado para analizar diversas estrategias sobre el gas natural. El modelo podrá mostrar cuantitativamente el efecto a largo plazo que cualquier decisión del Estado pudiera ejercer sobre cualquier dimensión del desarrollo sostenible del país.

[1] Word Energy Council. "Survey of Energy: Natural Gas", 2001. http://www.worldenergy.org/wec-geis/publications/reports/ ser/gas/gas.asp

[2] Forrester, J. "Industrial Dynamics", 1961.

[3] Ministerio de Energía y Minas, "Preguntas frecuentes en relación al Gas Natural", http://www.minem.gob.pe/archivos/dgh/publicaciones/Preguntas/preguntasgasnatural.pdf.

[4] Porter, M. "Ventaja competitiva, creación y sostenimiento de un desempeño superior", 1990.

Porter, M. "La ventaja competitiva de las naciones", 1991.

[5] Gurbaxani, V. "Conference competing via structure: Technology, pathership and value creation", Barcelona. Abril, 2005.

[6] UNESCO. "Intergovernmental Conference on the Administrative and Financial Aspects of Cultural Policies". Venice, 1970. 
[7] Energy Information Administration, "International Energy Outlook 2006", 2006. <http://tonto.eia.doe.gov/FTPROOT/forecasting/0484(2006).pdf >

[8] International Energy Agency, "World Energy Outlook 2004", 2004. http://www.iea.org/textbase/nppdf/free/2004/weo2004.pdf.

[9] Ministerio de Energía y Minas. "Ventajas del uso del gas natural sector industrial". <http://www.minem.gob.pe/archivos/dgh/publicaciones/gasnatural/gasindustrial.pdf $>$.

[10] Energy Information Administration, Country analysis briefs, "Natural Gas", <http://www.eia.doe.gov/emeu/cabs/Peru/ NaturalGas.html>.

[11] Comision Brundtland, Comision Mundial del Medio Ambiente y el desarrollo, 1987. <http://www.qed.econ.queensu.ca/pub/faculty/ keayi/ensc290slide3.pdf>

[12] Comisión Económica para América Latina y el Caribe (Cepal), "Energía y Desarrollo Sustentable en América Latina y el Caribe: Guía para la Formulación de Políticas Energéticas", 2003.

[13] Sunkel, O. "5th Intenational meeting of Intenational Society for ecological economics, Beyond Growth: Institutions and Policies for Sustainability". Chile, 1998.

[14] Consejo Mundial de energía. Reflexiones sobre la dinámica de los mercados de petróleo y gas natural, 2004.

$<$ http://www.worldenergy.org/wec-geis/global/downloads/statements/stat2004es.pdf >.

[15] Gonzáles del Valle, A. Consejo Nacional del Medio Ambiente Perú. "Informe final de estimación de la valoración económica de los impactos de la contaminación atmosférica por PTS y MN10 en la salud de la población de Lima Metropolitana". Noviembre, 2004.

[16] CEPAL. "La industria del gas natural en América del Sur: situación y posibilidades de integración de mercados". Diciembre, 2004. http://www.cte.org.pe/Infotec/GasNatural/pdf/Seminario08092005 /2.\%2OPLUSPETROL.pdf >

[17] CEPAL. "Estudio de suministro de gas natural desde Venezuela y Colombia a Costa Rica y Panamá". Junio, 2002.

[18] Martínez, E. Osinerg. "Seminario del Gas Natural en el Perú", Centrum PUCP. Setiembre, 2005. 
[19] Schneider, F. "The size and development of shadow economies and shadow economy labor force" - Shadow Economy, 2002. $<$ http://www.economics.uni-linz.ac.at/Schneider/Encyclopedia.PDF>

[20] Osinerg. Control de la informalidad en actividades relacionadas a hidrocarburos. <http://www.osinerg.gob.pe:8888/SPH/html/control_informalidad/default.htm>.

[21] Ministerio de Energía y Minas. Nota de prensa: "Sol a sol se pagará conversión de vehículos a gas natural". 2 de octubre, 2005. <http:// www.minem.gob.pe/common/prensa/data/notihoy1641.pdf $>$.

[22] Banco Interamericano de Desarrollo (BID). "La competitividad de Perú después de la década de reforma: Diagnóstico y propuestas", 2003. < http://www.ahciet.net/economia/macroeconomia/perufinanciero.pdf $>$

[23] Instituto Nacional de Estadística e Informática. "Proyecciones de la Población Perú 1995 -2025". <http://www.inei.gob.pe/biblioineipub/bancopub/Est/Lib0006/n00.htm>.

[24] Ministerio de energía y minas. "Ventas internas según tipo de combustible". Julio, 2006. <http://www.minem.gob.pe/archivos/dgh/ estadisticas/infor-mens/2006/agosto/venta.pdf >.

[25] CONAM. GEO Perú. "Informe nacional sobre el estado del ambiente", 2000. <http://www.conam.gob.pe/geo/ii31c.htm>.

[26] Ministerio de Transporte y Comunicaciones. "Estadísticas del 2006". $<$ http://www.mtc.gob.pe/portal/estadisticas/circulacion.asp > .

[27] United Nations Centre for Human Settlements (Habitat). "Cities in a globalizing world: Global report on human settlements 2001". London, Earthscan, 2001. <http://www.infoforhealth.org/pr/prs/ sm16/m16.pdf >.

[28] CEPAL. "Estrategia para el fomento de las fuentes renovables de energía en América Central". Agosto, 2004. 